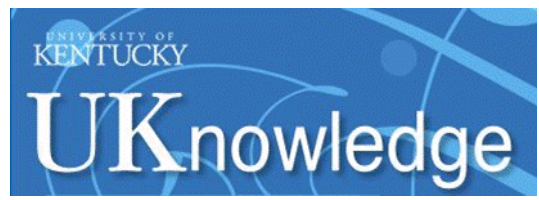

University of Kentucky

UKnowledge

\title{
Optimization of Aggregated EV Power in Residential Communities with Smart Homes
}

\author{
Huangjie Gong \\ University of Kentucky, huangjie.gong@uky.edu \\ Dan M. Ionel \\ University of Kentucky, dan.ionel@uky.edu
}

Follow this and additional works at: https://uknowledge.uky.edu/peik_facpub

Part of the Electrical and Electronics Commons, and the Power and Energy Commons

Right click to open a feedback form in a new tab to let us know how this document benefits you.

\section{Repository Citation}

Gong, Huangjie and Ionel, Dan M., "Optimization of Aggregated EV Power in Residential Communities with Smart Homes" (2020). Power and Energy Institute of Kentucky Faculty Publications. 2.

https://uknowledge.uky.edu/peik_facpub/2

This Conference Proceeding is brought to you for free and open access by the Power and Energy Institute of Kentucky at UKnowledge. It has been accepted for inclusion in Power and Energy Institute of Kentucky Faculty Publications by an authorized administrator of UKnowledge. For more information, please contact UKnowledge@lsv.uky.edu. 


\title{
Optimization of Aggregated EV Power in Residential Communities with Smart Homes
}

\author{
Digital Object Identifier (DOI) \\ https://doi.org/10.1109/ITEC48692.2020.9161532
}

Notes/Citation Information

Published in 2020 IEEE Transportation Electrification Conference \& Expo (ITEC).

(C) 2020 IEEE Copyright Notice. "Personal use of this material is permitted. Permission from IEEE must be obtained for all other uses, in any current or future media, including reprinting/republishing this material for advertising or promotional purposes, creating new collective works, for resale or redistribution to servers or lists, or reuse of any copyrighted component of this work in other works."

The document available for download is the authors' manuscript version accepted for publication. The final published version is copyrighted by IEEE and is available as: Gong, H., and lonel, D. M., "Optimization of Aggregated EV Power in Residential Communities with Smart Homes", 2020 IEEE Transportation Electrification Conference \& Expo (ITEC), Chicago, IL, USA, 2020, pp. 779-782, doi: 10.1109/ ITEC48692.2020.9161532 


\title{
Optimization of Aggregated EV Power in Residential Communities with Smart Homes
}

\author{
Huangjie Gong, Student Member, IEEE, and Dan M. Ionel, Fellow, IEEE \\ SPARK Lab, Department of Electrical and Computer Engineering, University of Kentucky, Lexington, KY, USA \\ huangjie.gong@uky.edu,dan.ionel@ieee.org
}

\begin{abstract}
Electric vehicles (EVs) tend to increase peak power for residences in the evening when house owners return home and begin charging. The aggregated EV charging demand can cause a sudden rise in the peak power at the distribution system level, resulting in a "dragon curve". Such phenomenon, combined with the "duck curve" that is caused by high photovoltaic (PV) penetration in residential communities, requires fast ramping rates and expanded capabilities for local distribution transformers and main feeder cables provided by the utility. As a solution, a residential energy storage system (RESS) can store surplus PV generation during midday and use the stored energy to support the peak power demand in the evening. House owners benefit from this strategy by avoiding electricity sales to the grid at low rates and by reducing energy usage during high Time-of-Use (ToU) periods. In this paper, a community with smart homes that include PV systems, RESSs and EVs was modeled. The EV models were developed based on data from the National Travel Household Survey (NHTS). The EV charging and RESS operation were scheduled to reduce the daily utility charge. The entire power system worked as virtual power plant as it kept the aggregated power constant for a long period of time.
\end{abstract}

Index Terms - Smart Home, Electric Vehicle (EV), Residential Energy Storage System (RESS), Residential Distribution Power System, Dragon Curve, NHTS.

\section{INTRODUCTION}

The increasing penetration of electric vehicles (EVs) in residential communities has created a phenomenon known as the "dragon curve". Many EVs begin charging at around the same time in the evening as house owners return home. Rooftop photovoltaic (PV) for residences can provide electricity to charge these EVs, but the mismatch between high PV generation and EV charging times results in an even more severe "duck curve" at the distribution power system level. Time-of-Use (ToU) rates are one of the efforts that helps to guide the behaviors of house electricity usage [1]. For an individual house, a residential energy storage system (RESS) is essential to realize the response for different price signals.

The distribution power system can be modeled by software such as GridLAB-D, OpenDSS or MATPOWER. GridLAB$D$ provides an integrated power system model that includes the residences. Each residence can be further defined with details for an inverter, a PV system, an EV, or a RESS [2]. The power system can be modeled by OpenDSS at the community distribution level and with EnergyPlus at the residential level. Efforts have been made to realize the dynamic communication between OpenDSS and multiple EnergyPlus threadings [3].
The data from the National Travel Household Survey (NHTS), which was most recently updated in 2017, has been used in the past for the modeling of EV charging power [4]. Even though the random nature of human behavior dominates the individual EV performance, the aggregated EV charging load is subject to probability distributions according to the data. Previous research has modeled the aggregated EV charging load based on EV arrival time, arrival SOC, and charging finish time [5], [6].

In this paper, a residential community of 1,000 homes with combinations of PV systems, RESSs, and EVs was modeled. The EV charging power for the homes was modeled based on the NHTS 2017 data. A ToU rate was applied to calculate the utility charge for the studied day. EV charging and operation of RESS were scheduled. The impacts due to different penetrations of RESS and PV on the power system level were studied.

\section{SySTEM Modeling}

The proposed system comprises of residences with PV systems, RESSs, and EVs (Fig. 1). The residential load can be generated by GridLAB-D. Solar power can be calculated based on given weather data. It should be noted that the residential load might be negative due to the generators in the smart homes. This will reverse the direction of power flow.

The EV charging loads in the distribution system were decided by three sets of variables: charging finish time, arrival time, and arrival SOC. In this study, the charging finish time was set to 6:00 in the morning. The arrival time and arrival SOC were estimated from the NHTS 2017 data.

The arrival time is categorized into 15-minute intervals from the NHTS 2017 data. Its probability density function (PDF) was estimated using the kernel density estimation (KDE). With this, the distribution of arrival times for 1,000 EVs was generated (Fig. 2). The KDE function is defined as follows,

$$
\widehat{f}(t ; h)=\frac{1}{N} \sum_{i=1}^{N} K\left(t-t_{i} ; h\right),
$$

where $\widehat{f}(t ; h)$ is the estimated $\mathrm{KDE} ; K$, the kernel function, which is triangular in the study. The bandwidth was set to 0.2 in the study.

The daily driving distance from the NHTS 2017 data was used to estimate the arrival SOC for the EVs. The distribution of daily driving distance less than 120 miles was shown (Fig. 


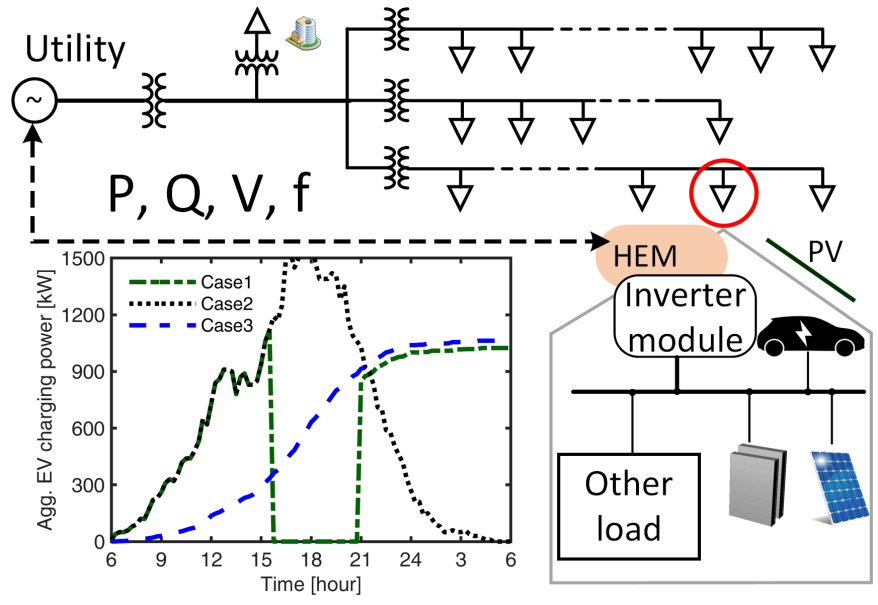

Figure 1. The scheme for the distribution system modeling. Residences with PVs, EVs, and RESSs were considered. Other loads and PV data were obtained with measured data from local utilities.

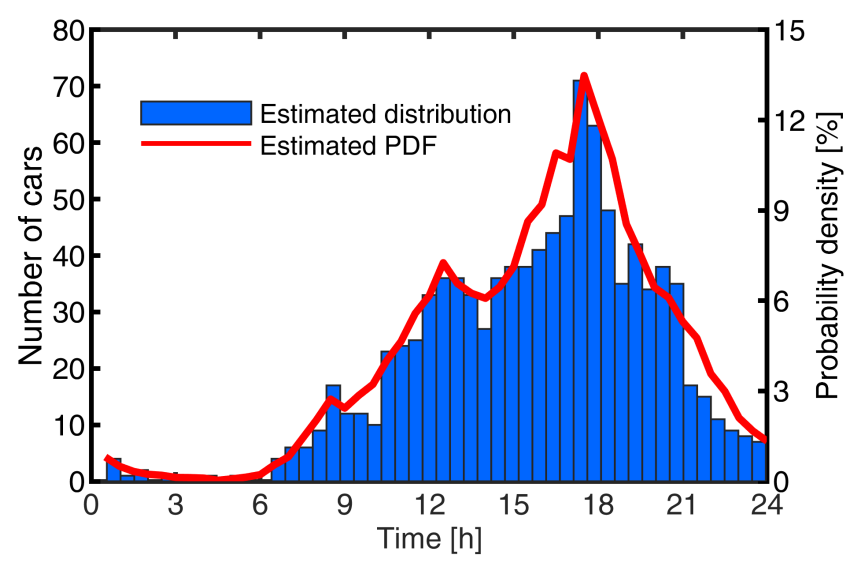

Figure 2. Distribution of time when EVs arrive home. The PDF was calculated by the KDE based on NHTS 2017 data. The histogram is the estimated distribution for 1,000 EVs.

3). The distribution was fit as a lognormal distribution [7], as follows,

$$
f(d)=\frac{1}{d \sigma \sqrt{2 \pi}} \exp \left(-\frac{(\ln d-\mu)^{2}}{2 \sigma^{2}}\right),
$$

where $d$ is the driving distance, the mean of lognormal distribution $\mu=3$; and the deviation $\sigma=1.12$ in the study.

The PDF of arrival SOC for EVs is related to the daily driving distance and was described as follows,

$$
f\left(S O C_{i}^{a}\right)=\left(1-\frac{d}{d_{M}}\right) \times 100 \%,
$$

where $d_{M}$ is the maximum driving distance allowed by the EV battery energy capacity. In the study, The effective cost per mile was set to 0.46 [8]. Assuming that each EV battery energy capacity is $90 \mathrm{kWh}$, the $d_{M}$ used in the study was 196 miles. The estimated PDF and distribution of arrival SOC for 1,000 EVs are shown (Fig. 4).

The individual EV power was calculated with the arrival time, arrival SOC, and charging finish time. The energy

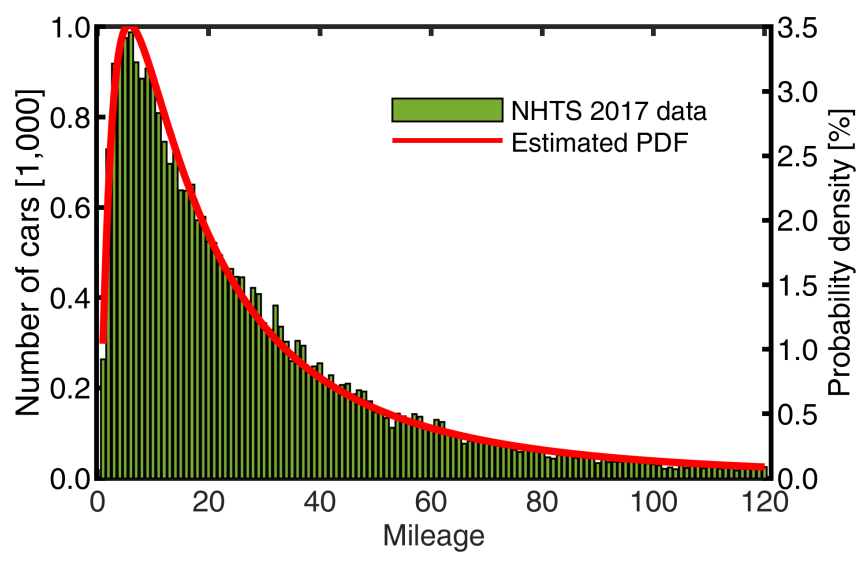

Figure 3. Distribution of daily mileage for EVs based on NHTS 2017 data. Daily driving distances of more than 120 miles are excluded in the study. The PDF for the distribution is described as a lognormal function.

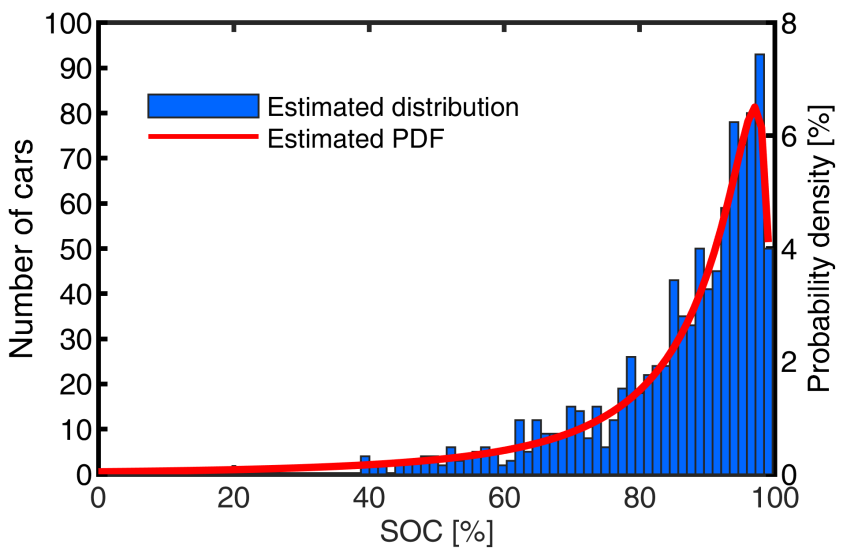

Figure 4. Distribution of SOCs for EVs when they arrive home. The PDF was calculated based on the KDE of daily mileage. The histogram is the estimated distribution for 1,000 EVs.

required for an $\mathrm{EV}$ is subject to the following,

$$
\int_{t_{a, i}}^{t_{f, i}} P_{E, i}^{t} d t=E_{i}-E_{i} \times S O C_{a, i},
$$

where $t_{a, i}$, the arrival time subject to $(1) ; t_{f, i}$, charging finish time which was fixed to 6:00 in the morning; $P_{E, i}^{t}$, EV charging power of house i at time $\mathrm{t}$; $E_{i}$, the EV battery energy capacity; $S O C_{a, i}$, arrival SOC subject to (3).

\section{EV AND RESS SCHEDULING}

The main objective for the study is to minimize the daily utility charge for electricity usage of the entire distribution system, described as follows,

$$
\operatorname{Min} \sum_{1}^{n} U C^{t},
$$

where $U C^{t}$ is the utility charge for the entire distribution power system at time step $t$.

The utility charge at time step $t$ is calculated as,

$$
U C^{t}=\int\left(E_{b}^{t} \cdot r_{b}^{t}-E_{s}^{t} \cdot r_{s}^{t}\right) d t,
$$




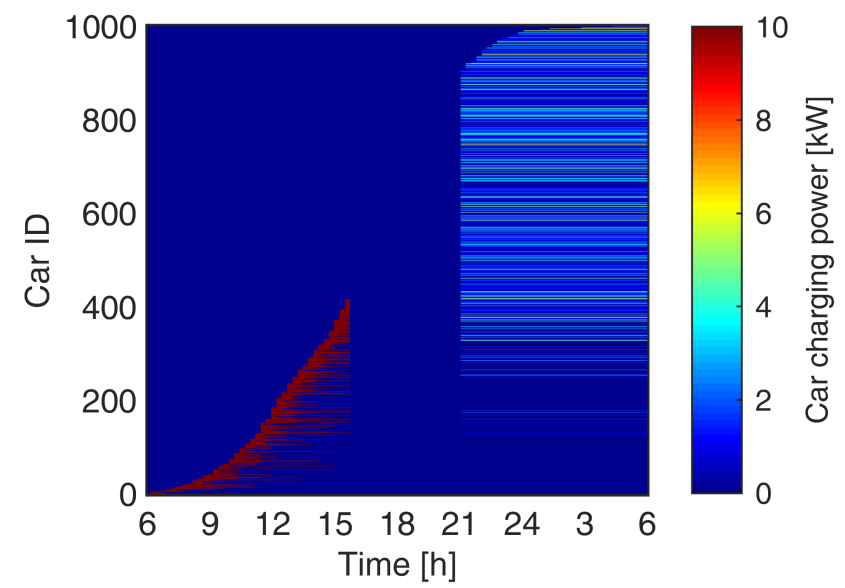

Figure 5. The charging power for all EVs in the distribution system. The EVs were charged at maximum and minimum power before and after the high ToU time, respectively. During the high ToU time, no EVs were charged.

where $E_{b}^{t}$ is energy purchased from the utility at time $\mathrm{t} ; r_{b}^{t}$, utility charge rate at time $\mathrm{t}$; $E_{s}^{t}$, energy sold to the utility at time $\mathrm{t} ; r_{s}^{t}$, the rate utility pays to buy back energy at time $t$.

The constrains include the power balance of the community,

$$
P_{G}^{t}=P_{P V}^{t}+P_{E}^{t}+P_{R E S S}^{t}+P_{R}^{t},
$$

where $P_{G}^{t}$ is the power purchased from the grid; $P_{P V}^{t}$, the PV generation; $P_{E}^{t}$, the charging power of EVs; $P_{R E S S}^{t}$, the charging power of RESSs; $P_{R}$, other residential power usage.

The energy capacity for RESSs is described as,

$$
20 \% \leqslant S O C_{R E S S}^{t} \leqslant 100 \% .
$$

The EV and RESS power values are defined as independent variables with their ranges given as follows,

$$
\begin{gathered}
P_{E, i}^{t} \leqslant P_{E, i, \max }, \\
\left|P_{R E S S}^{t}\right| \leqslant N \cdot P_{R E S S, \max },
\end{gathered}
$$

where $P_{E, i, \max }$, the maximum charging power of $\mathrm{EV}$ in house i; $N$, house numbers; $P_{R E S S, \max }$, the rated power for an individual RESS.

The ToU electricity rate indicates that most EVs arrive home during the peak charging period [1]. Therefore, the charging of EVs must avoid the high ToU rates to minimize utility charges. EVs that were available when there was surplus PV generation were charged with maximum power to consume the local solar power. EVs were charged with minimum power after the high ToU period. The EV scheduling is described as follows,

$$
P_{E, i}^{t}= \begin{cases}P_{E, i, \max }, & 1 \leq t \leq \mathrm{ToU}_{s} \\ 0, & T o U_{s} \leq t \leq \mathrm{ToU}_{e} \\ P_{E, i, \min }, & t \leq n,\end{cases}
$$

where $T o U_{s}, T o U_{e}$ stand for high ToU start and end time, respectively; $P_{E, i, \min }$ is the minimum power required to charge EV at house $i$ to $100 \%$ at the last time step. The individual EV charging power is shown (5). EVs were charged to $100 \%$ SOC at 6:00 the next morning (Fig. 6).

The RESS of the distribution system was considered as a

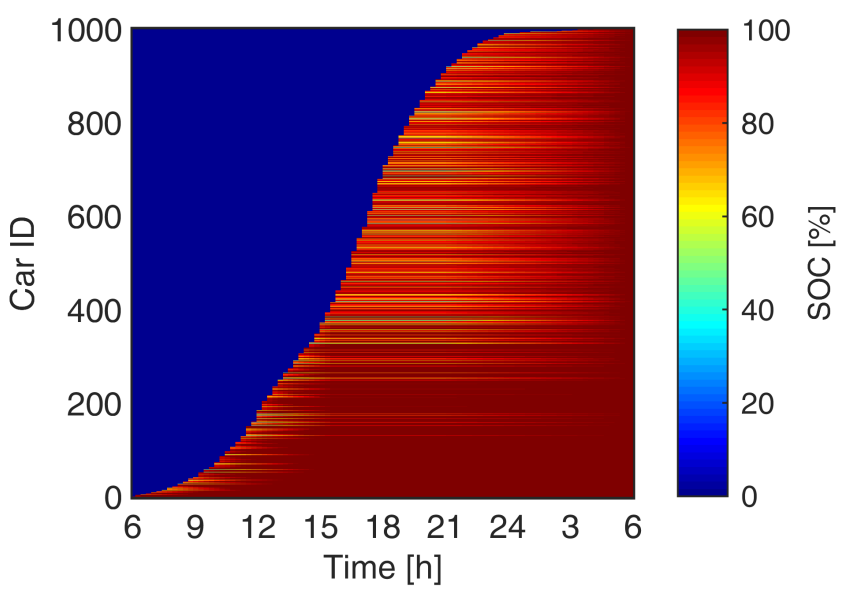

Figure 6. The SOCs for all EVs in the distribution system. The SOCs were regarded as 0 before EVs arrived home. EVs were guaranteed 100\% SOC at 6:00 the next day.

large battery. It started with a SOC of 50\%, was charged to $100 \%$ before high ToU period, discharged to $20 \%$ during the high ToU period, and recharged to $50 \%$ afterwards, as follows,

$$
\begin{cases}\int_{1}^{T o U_{s}} P_{R E S S}^{t} d t=50 \%, & 1 \leq t \leq T o U_{s} \\ \int_{T o U_{e}}^{T o U_{s}} P_{R E S S}^{t} d t=-80 \%, & T o U_{s} \leq t \leq T o U_{e} \\ \int_{T o U_{e}}^{n} P_{R E S S}^{t} d t=30 \%, & t \leq n .\end{cases}
$$

\section{CAse Studies}

The residential community being studied had 1,000 houses, each of which with a single EV. The residential load was from one typical summer day in Glasgow, KY and averaged to represent 1,000 homes [9]. The PV data is from LG\&E KU and was scaled to the ratings of $5 \mathrm{~kW}$ for each house [10]. The RESS is rated $9.8 \mathrm{kWh} / 5 \mathrm{~kW}$ and started with a SOC of $50 \%$. The EV SOCs and arrival time are subject to the distribution (Fig. 4 and Fig. 2). The EVs were not allowed to discharge in the study. The resolution for the study was 1-minute.

The aggregated power of EVs, RESSs, and net metered power for the residential distribution system on the example summer day are shown (Fig. 7). In this case all of the houses had a PV system and a RESS. The EVs were only charged when the ToU is not high (Fig. 5). The RESSs were charged when the PV generation was high and discharged during the high ToU period. After high ToU period, the RESSs were charged to $50 \%$. The proposed schedule reduced the electricity usage during the high ToU time, therefore, it reduced the total utility charge for the entire community. The entire residential community also served as a virtual power plant (VPP) for coordination of EVs and RESSs. As a VPP, the system managed to keep net metered power stable for a long period of time.

Simulation results for residential communities with different penetrations of EVs and RESSs are shown (Fig. 8). The 0\% curve represents the baseline case without PVs or RESSs where the optimal scheduling of EVs avoided the high ToU. With the increase in PV penetration, solar generation became 


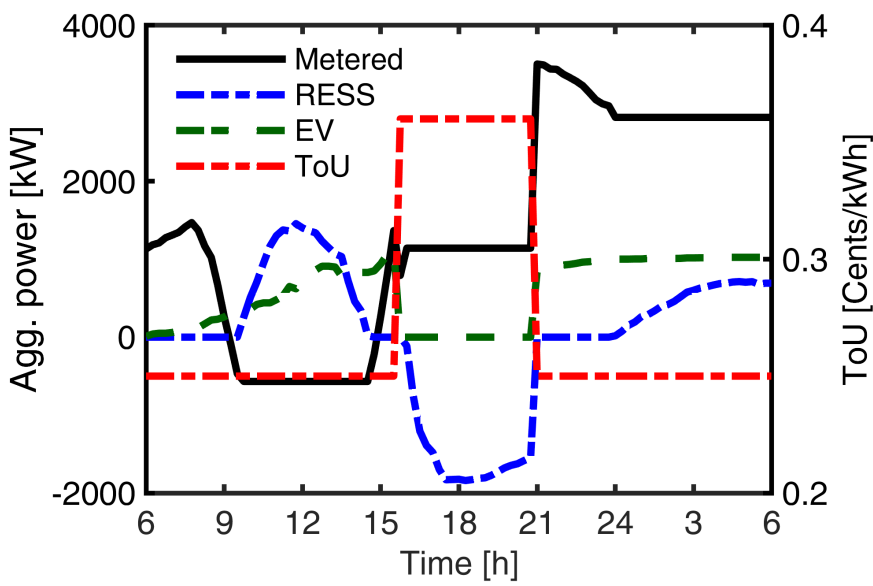

Figure 7. The aggregated power at distribution level. The EV curve was decided by the charging schedule. The RESS was first charged when the PV generation is high, discharged during high ToU period, and was charged to $50 \%$ afterwards. The metered curve shows that the community sold less electricity to the utility when PV generation was high and reduced usage during the high ToU period.

higher. In the case of $100 \%$, houses had to sell electricity back to the utility in the afternoon.

Higher RESS penetration means larger total capacity. Therefore, the higher the penetration, the lower the aggregated net metered power during the high ToU period, and the lower the utility charge. The averaged total cost per house for $0 \%-$ $100 \%$ are $\$ 17, \$ 15, \$ 13, \$ 11, \$ 10$, respectively. The $100 \%$ penetration has the advantage of peak reduction during high ToU time. The high solar generation also means that residences must sell electricity back to the grid at a low price. Charging of RESSs after high ToU time did not increase the peak demand.

\section{Conclusion}

A community with 1,000 houses was studied in this paper. The residential load and PV generation data were from the experimental data in a typical summer day. The electricity for the entire community was charged based on a ToU rate. The scheduling for EV charging and RESS operation were proposed to reduce the daily utility charge. Results show that the entire residential community worked as a VPP under the proposed schedule.

The distribution of arrival time and daily driving mileage were estimated according to NHTS 2017 data. The arrival SOCs for EVs were calculated based on the daily driving mileage. An EV charging schedule was proposed in which the high ToU period was avoided and a $100 \%$ SOC was guaranteed at 6:00 in the morning on the next day.

The influences of different penetrations for RESSs and PVs on the aggregated power and daily utility charge were studied. Simulation results show that the coordination of RESSs and EVs resulted in the reduction of daily utility charge. The entire residential community can work as a VPP under the proposed schedule for RESSs and EVs.

\section{ACKNOWLEDGMENT}

This research was supported in part by the DE-EE0008352

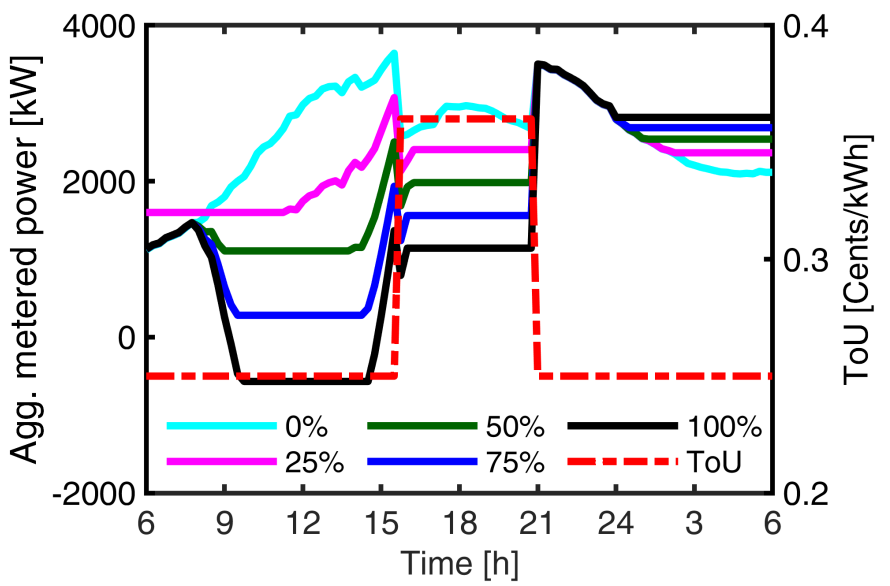

Figure 8. Metered powers for different penetrations of EVs and RESSs under optimal schedules. Percentage shows the penetration of residences with a PV system and RESS. The $0 \%$ case stands for the baseline. Higher penetration leads to more PV generation in the afternoon and lower electricity usage during the high ToU period.

project "Solar Power Electronics Modular Integrated Node Platform" led by FlexPower, Inc. and sponsored by the Department of Energy. The support of the University of Kentucky, the L. Stanley Pigman endowment, and of the SPARK program is also gratefully acknowledged.

\section{REFERENCES}

[1] "Time-of-Use (TOU) Rate Plans, Southern California Edison," https: //www.sce.com/residential/rates/Time-Of-Use-Residential-Rate-Plans, accessed: 2020-05-13.

[2] T. Markel, A. Meintz, K. Hardy, B. Chen, T. Bohn, J. Smart, D. Scoffield, R. Hovsapian, S. Saxena, J. MacDonald et al., "Multi-lab EV smart grid integration requirements study. providing guidance on technology development and demonstration," National Renewable Energy Lab.(NREL), Golden, CO (United States), Tech. Rep., 2015.

[3] H. Gong, V. Rallabandi, D. M. Ionel, D. Colliver, S. Duerr, and C. Ababei, "Dynamic modeling and optimal design for net zero energy houses including hybrid electric and thermal energy storage," IEEE Transactions on Industry Applications, 2020.

[4] "National Travel Household Survey," https://nhts.ornl.gov/, accessed: 2020-5-13.

[5] K. Park, S. Yoon, and E. Hwang, "Flexible charging coordination for plug-in electric vehicles based on uniform stochastic charging demand and time-of-use tariff," in 2019 IEEE Transportation Electrification Conference and Expo (ITEC). IEEE, 2019, pp. 1-4.

[6] B. Khaki, Y.-W. Chung, C. Chu, and R. Gadh, "Probabilistic electric vehicle load management in distribution grids," in 2019 IEEE Transportation Electrification Conference and Expo (ITEC). IEEE, 2019, pp. 1-6.

[7] Q. Liang, L. Lin, B. Zhou, and W. Zhao, "Modeling of pev charging load based on trip chain theory and the impact of pev on distribution networks," in 2018 International Conference on Power System Technology (POWERCON). IEEE, 2018, pp. 1789-1794.

[8] S. Shafiee, M. Fotuhi-Firuzabad, and M. Rastegar, "Investigating the impacts of plug-in hybrid electric vehicles on power distribution systems," IEEE Transactions on Smart Grid, vol. 4, no. 3, pp. 1351-1360, 2013.

[9] H. Gong, V. Rallabandi, M. L. McIntyre, and D. M. Ionel, "On the optimal energy controls for large scale residential communities including smart homes," in 2019 IEEE Energy Conversion Congress and Exposition (ECCE). IEEE, pp. 503-507.

[10] "Live Solar Generation Data, LG\&E KU," https://lge-ku.com/ live-solar-generation, accessed: 2020-5-13. 